\title{
Risk Factors and Biomarkers of Ischemic Stroke in Cancer Patients
}

\author{
Kwangsoo Kim, Ji-Hun Lee \\ Department of Neurology, Kosin University College of Medicine, Busan, Korea
}

Background and Purpose Stroke is common among cancer patients. However, risk factors and biomarkers of stroke in cancer patients are not well established. This study aimed to investigate risk factors and biomarkers as well as etiology of ischemic stroke in cancer patients. Methods A retrospective review was conducted in cancer patients with ischemic stroke who were admitted to a general hospital in Busan, Korea, between January 2003 and December 2012. The risk factors and biomarkers for stroke and stroke subtypes in cancer patients were compared with age- and sex-matched noncancer patients with ischemic stroke who were admitted to the same hospital during the same period.

Results One hundred fifty-six cancer patients with ischemic stroke were identified. Cancer patients with ischemic stroke were found to have a significantly lower proportion of hypertension, atrial fibrillation, hyperlipidemia, and ischemic heart disease than noncancer patients with ischemic stroke. However, stroke biomarkers, such as erythrocyte sedimentation rate and high-sensitivity C-reactive protein, fibrinogen, pro-brain natriuretic peptide, and D-dimer levels, were significantly increased in cancer patients with ischemic stroke than in noncancer patients. Large-artery atherosclerosis and stroke of undetermined cause were more common in cancer patients with ischemic stroke than in noncancer patients with ischemic stroke.

Conclusions Cancer patients with ischemic stroke showed different risk factors, stroke biomarkers, and stroke etiology compared with noncancer patients with ischemic stroke.

\author{
Correspondence: Kwangsoo Kim \\ Department of Neurology, Kosin \\ University College of Medicine, 262 \\ Gamcheon-ro, Seo-gu, Busan 602-703, \\ Korea \\ Tel: +82-51-990-6274 \\ Fax: +82-51-990-3077 \\ E-mail: nekim@ns.kosinmed.or.kr
}

Received: September 2, 2013

Revised: November 1, 2013

Accepted: November 1, 2013

The authors have no financial conflicts of interest.

Keywords Biomarker; Cancer; Ischemic stroke; Risk factor

\section{Introduction}

Cancer and ischemic stroke share many risk factors that lead to increased risk of stroke in cancer patients. A report of autopsies performed on cancer patients indicated that $14.6 \%$ patients had evidence of pathological cerebrovascular disease and $7.4 \%$ had clinical symptoms of stroke. ${ }^{1}$ However, only $3.5 \%$ of cancer patients sustained a stroke; these findings were similar with that of the general population. ${ }^{2}$

The causes of ischemic stroke in patients with and without cancer may be different. Cancer increases a patient's hypercoag- ulable states that increase deep vein thrombosis and nonbacterial thrombotic endocarditis. ${ }^{3}$ The pathogenesis of stroke, including the presence of a hypercoagulable state and increased D-dimer levels, an important factor, in most cancer patients is different from that of the general population. ${ }^{4,5}$ Other reports have stated that atherosclerosis and coagulopathy may be the dominant factors in the pathogenesis of stroke in cancer patients. ${ }^{2}$ Therefore, there has been dispute regarding the risk factors in the pathogenesis of stroke in cancer patients.

Stroke biomarkers can be used in understanding pathophysiology, screening high-risk patients, predicting clinical outcomes, 
and effective treatment of stroke patients. ${ }^{6}$ The biomarkers Ddimer, brain natriuretic peptide (BNP), fibrinogen, $\mathrm{C}$-reactive protein (CRP), and erythrocyte sedimentation rate (ESR) are significantly increased in patients with ischemic stroke. ${ }^{7,8}$ Moreover, increased D-dimer and BNP levels are associated with nonbacterial thrombotic endocarditis, and increased ESR is associated with lacunar and atherothrombotic stroke. ${ }^{7,8}$

The aim of this study was to investigate risk factors, biomarkers, and etiology of ischemic stroke in cancer patients by comparing them with age- and sex-matched noncancer patients with ischemic stroke.

\section{Methods}

\section{Patient selection}

The target group comprised cancer patients with ischemic stroke who were admitted to a general hospital in Busan, Korea, between January 2003 and December 2012. The cancer ischemic stroke group comprised patients with active cancer prior to the onset of ischemic stroke. This study did not consider the duration between cancer diagnosis and the onset of the ischemic event. The control group comprised age- and sex-matched noncancer patients with ischemic stroke who were admitted to the same hospital during the same period. The control group patients were selected at random.

\section{Data collection}

A retrospective review of cancer patients with ischemic stroke was performed to identify the cancer types, metastasis, stroke subtypes, stroke risk factors, and clinical and laboratory results. Hypertension, hyperlipidemia, atrial fibrillation, ischemic heart disease, smoking, alcohol intake, and past individual and family history of stroke were established as risk factors of stroke. ESR, high-sensitivity CRP (hs-CRP), fibrinogen, pro-BNP, D-dimer, and homocysteine were established as biomarkers of stroke.

\section{Definition of stroke risk factors}

Hypertension was established when systolic blood pressure was $>140 \mathrm{mmHg}$ and diastolic blood pressure was $>90 \mathrm{mmHg}$ at rest, or if the patient was taking hypertension medication. $\mathrm{Di}$ abetes was established when fasting glucose level was $>126$ $\mathrm{mg} / \mathrm{dL}$, postprandial 2-h glucose level was $>200 \mathrm{mg} / \mathrm{dL}$, or if the patient was taking medication for diabetes. Hyperlipidemia was established when one or more of the following levels were elevated: total cholesterol level, $>240 \mathrm{mg} / \mathrm{dL}$; low-density lipoprotein cholesterol level, $>130 \mathrm{mg} / \mathrm{dL}$; or triglyceride level $>200 \mathrm{mg} / \mathrm{dL}$. Atrial fibrillation was confirmed via an electrocardiogram taken at patient admission. Smoking was established when the patient had a past history of $\geq 5$ cigarettes per day. Alcohol intake was established with binge drinking or intake of $\geq 1$ times per week, irrespective of the amount consumed.

\section{Stroke biomarkers}

In order to examine biomarkers, blood was collected from patients with ischemic stroke during the acute ischemic event. Stroke biomarker concentrations were determined by assessing patient serum. Erythrocyte sedimentation rate was measured via photometrical capillary stopped flow kinetic analysis; hsCRP level was measured via latex-enhanced immunoturbidimetric analysis; fibrinogen level was measured via clotting assay based on viscosity; D-dimer level was measured via immunoturbidimetry; and homocysteine level was measured via direct chemiluminescent immunoassay.

\section{Stroke subtypes}

Brain magnetic resonance imaging, magnetic resonance angiography, transcranial Doppler ultrasonography, carotid ultrasonography, electrocardiography, and echocardiography were performed on most patients admitted with ischemic stroke. Stroke etiology was based on the TOAST classification ${ }^{9}$ and was classified as large-artery atherosclerosis, cardioembolism, small artery occlusion, and other determined and undetermined etiologies.

\section{Statistical analysis}

All data were analyzed using SPSS software (version 17.0; SPSS Inc., Chicago, IL). The Chi-square test was used to compare stroke risk factors and subtypes between cancer patients with ischemic stroke and the control group. Student's t-test was used to compare biomarkers between the two groups. Statistical significance was based on a $P$ value of $<0.05$.

\section{Results}

\section{Clinical characteristics}

The study included 156 (103 [66\%] male; 53 [34\%] female) cancer patients with ischemic stroke. Patient age ranged from 40 to 89 years, with an average of $70.3 \pm 9.8$ years. The control group also comprised 103 male and 53 female noncancer patients, with their age ranging from 40 to 89 years, and a mean age of $70.3 \pm 9.8$ years. The types of primary cancer in patients with ischemic cancer are listed in Table 1; the incidences (the descending order) of these types are as follows: stomach cancer in $33(21.2 \%)$ patients, lung cancer in 25 (16\%), colon cancer in 22 (14.1\%), and hepatobiliary cancer in 20 (12.8\%). Metastasis was present in $84(53.8 \%)$ patients. 
Table 1. Clinical Characteristics of Cancer Patients with Ischemic Stroke $(\mathrm{N}=156)$

\begin{tabular}{lc}
\hline Patient demographics & \\
\hline Age (year), mean \pm SD (range) & $70.3 \pm 9.8(40-89)$ \\
Gender (male/female), $\mathrm{n}(\%)$ & $103 / 53(66.0 / 34.0)$ \\
Cancer type, $\mathrm{n}(\%)$ & \\
Gastric & $33(21.2)$ \\
Lung & $25(16.0)$ \\
Colorectal & $22(14.1)$ \\
Hepatobiliary & $20(12.8)$ \\
Prostatic & $8(5.1)$ \\
Breast & $7(4.5)$ \\
Pancreatic & $7(4.5)$ \\
Cervical & $5(3.2)$ \\
Renal & $5(3.2)$ \\
Bladder & $4(2.6)$ \\
Hematologic & $4(2.6)$ \\
Other & $16(10.3)$ \\
Systemic cancer metastasis, $\mathrm{n}(\%)$ & $84(53.8)$ \\
\hline
\end{tabular}

Table 2. Stroke Risk Factors in Cancer vs Noncancer Patients with Ischemic Stroke

\begin{tabular}{lccr}
\hline Risk factors & $\begin{array}{c}\text { Cancer patients } \\
(\mathrm{n}=156)\end{array}$ & $\begin{array}{c}\text { Noncancer patients } \\
(\mathrm{n}=156)\end{array}$ & Pvalue $^{*}$ \\
\hline Age, year (mean \pm SD) & $70.3 \pm 9.8$ & $70.3 \pm 9.8$ & 1.00 \\
Male, $\mathrm{n}(\%)$ & $103(66.0)$ & $103(66.0)$ & 1.00 \\
Hypertension, $\mathrm{n}(\%)$ & $85(54.5)$ & $123(78.8)$ & $<0.01$ \\
Diabetes mellitus, $\mathrm{n}(\%)$ & $54(34.6)$ & $53(34.0)$ & 0.91 \\
Smoking, $\mathrm{n}(\%)$ & $28(17.9)$ & $34(21.8)$ & 0.40 \\
Drinking, $\mathrm{n}(\%)$ & $22(14.1)$ & $30(19.2)$ & 0.22 \\
Atrial fibrillation, $\mathrm{n}(\%)$ & $15(9.6)$ & $36(23.1)$ & $<0.01$ \\
Ischemic heart disease, $\mathrm{n}(\%)$ & $15(9.6)$ & $30(19.2)$ & 0.02 \\
Previous stroke, $\mathrm{n}(\%)$ & $14(9.0)$ & $27(17.3)$ & 0.12 \\
Hyperlipidemia, $\mathrm{n}(\%)$ & $10(6.4)$ & $62(39.7)$ & $<0.01$ \\
Family history, $\mathrm{n}(\%)$ & $0(0)$ & $5(3.2)$ & 0.02 \\
\hline
\end{tabular}

${ }^{*}$ Statistics were analyzed by Chi-square test.

\section{Comparison of stroke risk factors}

The risk factors of ischemic stroke in cancer patients, in descending order of incidence, are as follows: hypertension, diabetes, smoking, alcohol intake, atrial fibrillation, ischemic heart disease, past history of stroke, and hyperlipidemia. Compared with the noncancer patients with ischemic stroke, the incidences of these risk factors were statistically lower in hypertension $(P<0.01)$, atrial fibrillation $(P<0.01)$, hyperlipidemia $(P<0.01)$, ischemic heart disease $(P=0.02)$, and family history of stroke $(P=0.02)$ (Table 2).

\section{Comparison of stroke biomarkers}

The stroke biomarkers ESR and hs-CRP, fibrinogen, pro$\mathrm{BNP}$, and $\mathrm{D}$-dimer levels were statistically higher in cancer patients with ischemic stroke (all $P<0.01$ ) than in noncancer patients, whereas no difference was found in the homocysteine
Table 3. Stroke Biomarkers in Cancer vs Noncancer Patients with Ischemic Stroke

\begin{tabular}{lccr}
\hline Biomarker & $\begin{array}{c}\text { Cancer patients } \\
(\mathrm{n}=156)\end{array}$ & $\begin{array}{c}\text { Noncancer patients } \\
(\mathrm{n}=156)\end{array}$ & $P$ value* \\
\hline ESR $(\mathrm{mm} / \mathrm{h})$ & $31.3 \pm 28.5$ & $18.1 \pm 18.4$ & $<0.01$ \\
hs-CRP $(\mathrm{mg} / \mathrm{dL})$ & $5.0 \pm 6.9$ & $1.8 \pm 3.7$ & $<0.01$ \\
Fibrinogen $(\mathrm{mg} / \mathrm{dL})$ & $344.4 \pm 182.2$ & $311.9 \pm 103.9$ & $<0.01$ \\
pro-BNP $(\mathrm{pg} / \mathrm{mL})$ & $1,261.2 \pm 3.2$ & $531.6 \pm 1.0$ & $<0.01$ \\
D-dimer $(\mu \mathrm{g} / \mathrm{L})$ & $2,370.5 \pm 11,318.1$ & $324.2 \pm 439.5$ & $<0.01$ \\
Homocysteine $(\mu \mathrm{mol} / \mathrm{L})$ & $14.0 \pm 6.0$ & $15.0 \pm 7.1$ & 0.55 \\
\hline
\end{tabular}

Values are presented as mean \pm SD.

*Statistics were analyzed using Student's t-test.

ESR, erythrocyte sedimentation rate; hs-CRP, high-sensitivity C-reactive protein; proBNP, pro-brain natriuretic peptide; SD, standard deviation.

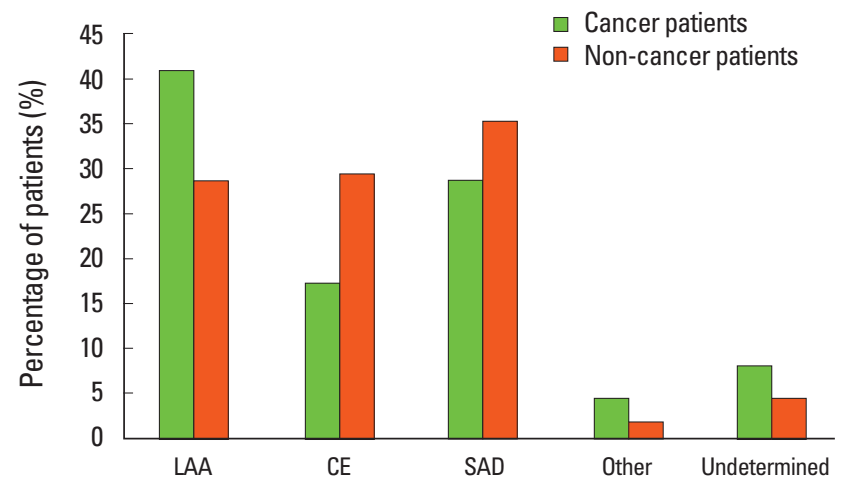

Figure 1. Stroke subtype in cancer versus noncancer patients with ischemic stroke. CE, cardioembolism; LAA, large artery atherosclerosis; Other, other determined etiology; $S A D$, small artery disease; Undermined, undetermined etiology.

levels between the groups (Table 3).

\section{Comparison of stroke etiology}

The different etiologies according to ischemic stroke subtypes in cancer patients, in descending order of incidence, are as follows: large-artery atherosclerosis (41\%), small artery occlusion (28.8\%), cardioembolism (17.3\%), undetermined etiology (8.3\%), and other determined etiology (4.5\%). The incidences of large-artery atherosclerosis and undetermined etiology were higher, whereas those of cardioembolism and small artery occlusion were lower in cancer patients than in noncancer patients $(P=0.02)$ (Figure 1).

\section{Discussion}

The incidences of hypertension, atrial fibrillation, hyperlipidemia, and ischemic heart disease were significantly lower in cancer patients with ischemic stroke than in noncancer patients with ischemic stroke. In contrast, biomarkers such as ESR and hs-CRP, fibrinogen, pro-BNP, and D-dimer levels were signifi- 
cantly increased in cancer patients with ischemic stroke.

Smoking is a common risk factor in both ischemic stroke and cancer and is also the etiology for most ischemic strokes in cancer patients. ${ }^{2,10}$ Most reports have found that hypertension and smoking are the most common causes of ischemic stroke in cancer patients. Additional causes include diabetes, ischemic heart disease, hyperlipidemia, alcohol intake, and atrial fibrillation. ${ }^{10-14}$ This study compared noncancer and cancer patients with ischemic stroke and found that the cancer group had a lower incidence of hypertension, atrial fibrillation, hyperlipidemia, and ischemic heart disease. Previous reports ${ }^{10-12,14}$ have found no differences in common stroke risk factors between cancer and noncancer patients with ischemic stroke. However, other reports have found low incidences of hypertension and hyperlipidemia in cancer patients with ischemic stroke compared with those of the noncancer group. ${ }^{15,16}$ Moreover, no significant differences in hyperlipidemia and atrial fibrillation incidences have been reported, but a tendency for lower incidence in cancer patients with ischemic stroke. ${ }^{14}$ This study hypothesized that in addition to the commonly known stroke risk factors, other factors may also be involved in cancer patients with ischemic stroke.

This study found that stroke biomarkers such as ESR and hs$\mathrm{CRP}$, fibrinogen, pro-BNP, and D-dimer levels were significantly increased in cancer patients compared with noncancer patients with ischemic stroke.

$\mathrm{D}$-dimer, a fibrin degradation product that is directly related to coagulation and fibrinolysis, is a marker for hypercoagulability. ${ }^{17}$ Its levels are increased in acute, subacute, and chronic ischemic stroke. ${ }^{7}$ Compared with noncancer patients with ischemic stroke, cancer patients with ischemic stroke have statistically significant higher levels of D-dimer, ${ }^{15,16}$ which coincides with our results. In previous studies, cancer patients with ischemic stroke (without common stroke risk factors) were found to have statistically significant higher levels, which corresponded with an increased incidence of embolic signal in transcranial Doppler monitoring; thus, it was hypothesized that coagulopathy and emboli in cancer patients may be the etiology for ischemic stroke. ${ }^{4,5,18}$

Fibrinogen is involved in thrombokinesis, platelet aggregation, blood viscosity, thrombus formation, and inflammation. Increase in fibrinogen concentrations is directly related to an increase in ischemic stroke incidence. ${ }^{19}$ Cancer patients with ischemic stroke have been found to have a statistically significant increased fibrinogen levels. ${ }^{8,20}$ No statistically significant difference was found in fibrinogen levels between cancer and noncancer patients with ischemic stroke; however, the cancer group had a tendency to have a higher fibrinogen levels. ${ }^{13}$ However, in the current study, cancer patients with ischemic stroke had a statistically significant higher level of fibrinogen than noncancer patients. Therefore, we hypothesize that the high fibrinogen levels may be because cancer is the primary disease.

Brain natriuretic peptide is a vasoactive peptide hormone that increases sodium excretion, urination, and vasodilation as well during the acute stage of ischemic stroke, and it is also a predictor of poor prognosis. ${ }^{7,21}$ It is increased in the cardioembolic subtype of ischemic stroke and is highly suggestive of cardioembolic etiology when serum concentrations are $>140 \mathrm{pg} / \mathrm{mL} .^{22}$ This study found that cancer patients with ischemic stroke had a statistically significant higher BNP levels. This study also found that even though noncancer patients with ischemic stroke had higher incidences of atrial fibrillation and ischemic heart disease, BNP concentrations were statistically higher in the cancer group with ischemic stroke. Therefore, we hypothesize that the high levels may be because cancer was the primary disease.

C-reactive protein is related to inflammation and progression of hemangioendotheliomal damage as an acute peptide, which increases in systemic inflammation and artherosclerotic disease. ${ }^{23}$ Its levels increase in acute ischemic stroke, and CRP is a useful aid in prognosis. ${ }^{7,8}$ No statistically significant difference was found in CRP levels between cancer and noncancer patients with ischemic stroke. ${ }^{13}$ On the other hand, we found that cancer patients with ischemic stroke had a statistically significant higher CRP concentrations. Therefore, we hypothesize that the high levels may be because cancer was the primary disease.

Erythrocyte sedimentation rate is a nonspecific marker of inflammation and infection. Inflammation activates coagulation, and a continued increase in ESR indicates progressive thrombogenesis and fibrinolysis in patients with stroke. In addition to being a predictor of poor prognosis, ${ }^{24}$ ESR is a surrogate marker for inflammation in small and large vasculopathies. Moreover, increases in ESR are related to artherosclerotic and lacunar stroke. ${ }^{8}$ This present study found that cancer patients with ischemic stroke had statistically significant higher ESRs. Therefore, we hypothesize that the high levels may be because of the presence of both cancer and ischemic stroke.

Homocysteine is an aminoacid byproduct of methionine metabolism, which aggravates artherosclerosis by damaging hemangioendothelial cells, increasing platelet aggregation through changing arachidonic acid metabolism, and decreasing anticoagulation factor activity. ${ }^{25}$ Increased homocysteine levels correlate with stenosis and occlusion of intracranial vessels in patients with ischemic stroke, and it also increases stroke risk. ${ }^{26,27}$ In the current study, no differences in homocysteine concentrations were found between cancer and noncancer patients with isch- 
emic stroke.

We found significantly higher levels of biomarkers such as ESR, hs-CRP, fibrinogen, pro-BNP, and D-dimer in the cancer with ischemic stroke group than those in the noncancer ischemic stroke group. These results imply a relationship between cancer patients and inflammation, thrombus formation, and coagulopathy, which could explain the increased frequency of ischemic stroke in the cancer patients compared with the noncancer patients.

The etiology in cancer patients with ischemic stroke consists of coagulopathies due to cytokine or microparticle-releasing cancer cells, intravascular coagulation or nonbacterial thrombotic endocarditis, occlusion due to metastasis, direct vascular compression due to metastasis or neurocarcinomas, coagulopathies due to chemotherapy or radiation therapy, vascular embolization (e.g., treatment of cancer vessels), and paraneoplastic causes. ${ }^{11,18,28}$ In previous studies, of the cancer patients with ischemic stroke, $40 \%$ did not have any etiology of general stroke patients, and these patients are thought to have closer relationship to an embolic etiology due to coagulopathies. ${ }^{4,18}$

This study found that etiology according to stroke subtypes in cancer patients with ischemic stroke consisted of (in descending order of incidence): large-artery atherosclerosis, small artery occlusion, cardioembolism, and undetermined etiology. The proportion of large-artery atherosclerosis and stroke of undetermined cause was more frequent in cancer patients than in noncancer patients with ischemic stroke. These results are different from those of past reports. Previous studies have reported that cancer patients with ischemic stroke have a higher incidence of thrombotic strokes (54\%), including cardioembolic strokes (15\%), than nonthrombotic strokes (46\%), including those due to undetermined causes (19\%), artherosclerotic causes (10\%), small-artery occlusion (12\%), and other determined causes (5\%), according to the TOAST classification..$^{10}$ Another report found that cancer patients with ischemic stroke have $39.8 \%$ difference in etiology compared with general stroke patients with artherosclerosis, cardioembolic, or lacunar stroke subtypes. ${ }^{4}$ It is also reported that $25.5 \%$ had a statistically significant higher incidence of not having a general stroke etiology in cancer patients with ischemic stroke compared to that of noncancer patients with ischemic stroke. ${ }^{13}$ However, stroke subtypes were similar in both cancer and noncancer patients with ischemic stroke. ${ }^{18}$ Stroke types according to the TOAST classification were not statistically significant between cancer and noncancer patients with ischemic stroke. ${ }^{14}$

The frequency of large-artery atherosclerosis as the etiology for ischemic stroke was significantly higher in cancer patients than in noncancer patients. We hypothesize that increased lev- els inflammatory biomarkers such as ESR, hs-CRP, and fibrinogen may be the reason for the increased frequency of large-artery atherosclerosis in ischemic stroke in cancer patients. Inflammatory processes play an important role in atherosclerosis development. ${ }^{29,30}$ The increased inflammation seen in cancer patients may be due to the increased frequency of large-artery atherosclerosis.

There are a few limitations to this study. First, the study group comprised patients who were admitted to a single hospital, which can lead to a selection bias. Second, the duration of cancer and that between cancer diagnosis and the onset of ischemic stroke were not considered. Third, cancer type, metastasis, and the effect of cancer treatment were not considered in this study. Further study may be needed to rectify these limitations.

\section{Conclusions}

Cancer patients with ischemic stroke demonstrated different risk factors, stroke biomarkers, and stroke etiology compared with noncancer patients with ischemic stroke. Therefore, it is recommended that in order to reduce the incidence of ischemic stroke in cancer patients, we should not only manage conventional risk factors, but also establish a distinct management of reducing inflammation and thrombus formation.

\section{References}

1. Graus F, Rogers LR, Posner JB. Cerebrovascular complications in patients with cancer. Medicine (Baltimore) 1985;64:16-35.

2. Chaturvedi S, Ansell J, Recht L. Should cerebral ischemic events in cancer patients be considered a manifestation of hypercoagulability? Stroke 1994;25:1215-1218.

3. Hiatt BK, Lentz SR. Prothrombotic States that Predispose to Stroke. Curr Treat Options Neurol 2002;4:417-425.

4. Kim SG, Hong JM, Kim HY, Lee J, Chung PW, Park KY, et al. Ischemic stroke in cancer patients with and without conventional mechanisms: a multicenter study in Korea. Stroke 2010; 41:798-801.

5. Seok JM, Kim SG, Kim JW, Chung CS, Kim GM, Lee KH, et al. Coagulopathy and embolic signal in cancer patients with ischemic stroke. Ann Neurol 2010;68:213-219.

6. Kim SJ, Moon GJ, Bang OY. Biomarkers for stroke. J Stroke 2013;15:27-37.

7. Montaner J, Perea-Gainza M, Delgado P, Ribó M, Chacón P, Rosell A, et al. Etiologic diagnosis of ischemic stroke subtypes with plasma biomarkers. Stroke 2008;39:2280-2287.

8. Alvarez-Perez FJ, Castelo-Branco M, Alvarez-Sabin J. Usefulness of measurement of fibrinogen, D-dimer, D-dimer/fibrin- 
ogen ratio, $\mathrm{C}$ reactive protein and erythrocyte sedimentation rate to assess the pathophysiology and mechanism of ischaemic stroke. J Neurol Neurosurg Psychiatry 2011;82:986-992.

9. Adams HP Jr, Davis PH, Leira EC, Chang KC, Bendixen BH, Clarke WR, et al. Baseline NIH Stroke Scale score strongly predicts outcome after stroke: a report of the Trial of Org 10172 in Acute Stroke Treatment (TOAST). Neurology 1999;53:126131.

10. Cestari DM, Weine DM, Panageas KS, Segal AZ, DeAngelis LM. Stroke in patients with cancer: incidence and etiology. Neurology 2004;62:2025-2030.

11. Grisold W, Oberndorfer S, Struhal W. Stroke and cancer: a review. Acta Neurol Scand 2009;119:1-16.

12. Stefan O, Vera N, Otto B, Heinz L, Wolfgang G. Stroke in cancer patients: a risk factor analysis. J Neurooncol 2009;94:221226.

13. Kim JM, Jung KH, Park KH, Lee ST, Chu K, Roh JK. Clinical manifestation of cancer related stroke: retrospective case-control study. J Neurooncol 2013;111:295-301.

14. Zhang YY, Chan DK, Cordato D, Shen Q, Sheng AZ. Stroke risk factor, pattern and outcome in patients with cancer. Acta Neurol Scand 2006;114:378-383.

15. Schwarzbach CJ, Schaefer A, Ebert A, Held V, Bolognese M, Kablau M, et al. Stroke and cancer: the importance of cancerassociated hypercoagulation as a possible stroke etiology. Stroke 2012;43:3029-3034.

16. Kono T, Ohtsuki T, Hosomi N, Takeda I, Aoki S, Sueda Y, et al. Cancer-associated ischemic stroke is associated with elevated D-dimer and fibrin degradation product levels in acute ischemic stroke with advanced cancer. Geriatr Gerontol Int 2012; 12:468-474.

17. Wilde JT, Kitchen S, Kinsey S, Greaves M, Preston FE. Plasma D-dimer levels and their relationship to serum fibrinogen/fibrin degradation products in hypercoagulable states. $\mathrm{Br} \mathrm{J} \mathrm{Hae-}$ matol 1989;71:65-70.

18. Bang OY, Seok JM, Kim SG, Hong JM, Kim HY, Lee J, et al. Ischemic stroke and cancer: stroke severely impacts cancer patients, while cancer increases the number of strokes. J Clin $\mathrm{Neu}$ - rol 2011;7:53-59.

19. Rothwell PM, Howard SC, Power DA, Gutnikov SA, Algra A, van Gijn J, et al. Fibrinogen concentration and risk of ischemic stroke and acute coronary events in 5113 patients with transient ischemic attack and minor ischemic stroke. Stroke 2004;35:23002305.

20. Prugger C, Luc G, Haas B, Morange PE, Ferrieres J, Amouyel P, et al. Multiple biomarkers for the prediction of ischemic stroke: the PRIME study. Arterioscler Thromb Vasc Biol 2013;33:659666.

21. Whiteley W, Wardlaw J, Dennis M, Lowe G, Rumley A, Sattar $\mathrm{N}$, et al. The use of blood biomarkers to predict poor outcome after acute transient ischemic attack or ischemic stroke. Stroke 2012;43:86-91.

22. Shibazaki K, Kimura K, Iguchi Y, Okada Y, Inoue T. Plasma brain natriuretic peptide can be a biological marker to distinguish cardioembolic stroke from other stroke types in acute ischemic stroke. Intern Med 2009;48:259-264.

23. Pasceri V, Willerson JT, Yeh ET. Direct proinflammatory effect of C-reactive protein on human endothelial cells. Circulation 2000;102:2165-2168.

24. Anuk T, Assayag EB, Rotstein R, Fusman R, Zeltser D, Berliner $S$, et al. Prognostic implications of admission inflammatory profile in acute ischemic neurological events. Acta Neurol Scand 2002;106:196-199.

25. Welch GN, Loscalozo J. Homocysteine and atherothrombosis. NEng J Med 1998;338:1042-1050.

26. Jeong SK, Seo JY, Cho YI. Homocysteine and internal carotid artery occlusion in ischemic stroke. J Atheroscler Thromb 2010; 17:963-969.

27. Perry IJ. Homocysteine and risk of stroke. J Cardiovasc Risk 1999; 6:235-240

28. Mulrooney DA, Blaes AH, Duprez D. Vascular injury in cancer survivors. J Cardiovasc Transl Res 2012;5:287-295.

29. Libby P. Inflammation in atherosclerosis. Nature 2002;420:868874.

30. Shishehbor MH, Bhatt DL. Inflammation and atherosclerosis. Curr Atheroscler Rep 2004;6:131-139. 